D. Niebur

Nagoya Math. J.

Vol. 52 (1973), 133-145

\title{
A CLASS OF NONANALYTIC AUTOMORPHIC FUNCTIONS
}

\author{
DOUGLAS NIEBUR
}

In this paper we consider a class of nonanalytic automorphic functions which were first mentioned to A. Selberg by C. L. Siegel. These functions have Fourier coefficients which are closely connected with the Fourier coefficients of analytic automorphic forms, and they are also eigenfunctions of the Laplace operator derived from the hyperbolic metric. We shall show how this latter property gives new results in the classical theory of automorphic forms.

I am indebted to Professor Selberg for introducing me to these functions.

\section{§1. Definitions and Notation}

Let $\Gamma$ be a discrete subgroup of $S L(2, R)$ having a fundamental domain of finite non-Euclidean area. We assume that $\Gamma$ has a single maximal parabolic subgroup $\Gamma_{\infty}=\left\{ \pm\left(\begin{array}{ll}1 & n \\ 0 & 1\end{array}\right): n \in Z\right\}$, although this assumption is not necessary. Let $\mathscr{D}$ be the fundamental domain containing the strip $S_{Y}=\{z: \operatorname{Im} z>Y, 0<\operatorname{Re} z<1\}$, for sufficiently large positive $Y$.

If $H$ denotes the upper half plane and $z$ and $z^{\prime}$ are in $H$, where $z=x+i y, z^{\prime}=x^{\prime}+i y^{\prime}$, then the non-Euclidean metric $d\left(z, z^{\prime}\right)$ is given by $\cosh d\left(z, z^{\prime}\right)=1+\left|z-z^{\prime}\right|^{2} / 2 y y^{\prime}$. The invariant measure is $d z=y^{-2} d x d y$ and the Laplace operator derived from the metric is

$$
D=y^{2}\left(\frac{\partial^{2}}{\partial x^{2}}+\frac{\partial^{2}}{\partial y^{2}}\right) \text {. }
$$

Let $L^{2}(\mathscr{D})$ be the space of all square-integrable automorphic functions $f$ defined on $H$; i.e., $f(M z)=f(z)$ for all $M \in \Gamma$ and $\int_{g}|f(z)|^{2} d z<\infty$. The inner product in this Hilbert space is denoted by $(f, g)=\int_{\mathscr{g}} f \bar{g} d z$, and the self-adjoint operator derived from $D$ is also denoted by $D$.

Received May 7, 1973. 
The Eisenstein series for $\Gamma$ is $E(z, s)=\sum_{M \in \Gamma_{\infty} \backslash \Gamma}(\operatorname{Im} M z)^{s}$ for $\operatorname{Re} s>1$. Because $D y^{s}=s(s-1) y^{s}$, it follows that $D E(z, s)=s(s-1) E(z, s)$. Also $E(M z, s)=E(z, s)$ for all $M \in \Gamma$. These properties are the main reasons why $E(z, s)$ has a meromorphic continuation to the entire $s$-plane.

As shown in Chapter II of [4], the Fourier expansion of the Eisenstein series is $E(x+i y, s)=\sum_{m=-\infty}^{\infty} a_{m}(y, s) e(m x)$, where $e(x)=\exp (2 \pi i x)$, $a_{0}(y, s)=y^{s}+\phi(s) y^{1-s}$, and $a_{m}(y, s)=a_{m}(s) y^{1 / 2} K_{s-1 / 2}(2 \pi|m| y)$ for $m \neq 0$. Here,

$$
\begin{aligned}
\phi(s) & =\Gamma\left(\frac{1}{2}\right) \Gamma\left(s-\frac{1}{2}\right) \phi_{0}(s) / \Gamma(s), \\
a_{m}(s) & =2 \pi^{s}|m|^{s-1 / 2} \phi_{m}(s) / \Gamma(s),
\end{aligned}
$$

and

$$
\phi_{m}(s)=\sum_{c>0} S(m, 0 ; c) c^{-2 s},
$$

where $S(m, n ; c)$ is the general Kloostermann sum $\sum_{0 \leq d<|c|} e((m a+n d) / c)$, $\left(\begin{array}{ll}a & * \\ c & d\end{array}\right) \in \Gamma$.

Although $\phi_{m}$ is defined by the Dirichlet series only for $\operatorname{Re} s>1$, the continuation of $E(z, s)$ results in the continuation of $\phi_{m}$. It is shown in [4] that $\phi(s) \phi(1-s)=1$ and $E(z, s)=\phi(s) E(z, 1-s)$. Thus, $a_{m}(s)=$ $\phi(s) a_{m}(1-s)$ since the Bessel function $K_{\nu}(y)$ is an even function of $\nu$.

We now come to the definition of the class of functions to which the title alludes. Suppose that $n$ is a nonzero integer and $s=\sigma+i t$, where $\sigma>1$. For $z \in H$, let

$$
F_{n}(z, s)=\sum_{M \in \Gamma_{\infty} \backslash \Gamma} e(n \operatorname{Re} M z)(\operatorname{Im} M z)^{1 / 2} I_{s-1 / 2}(2 \pi|n| \operatorname{Im} M z),
$$

where $I_{s-1 / 2}$ is the modified Bessel function of the first kind. This sum converges absolutely and uniformly on any strip of the form $\{x+i y$ : $|x| \leq A, y \geq \varepsilon>0\}$, because $y^{1 / 2} I_{s-1 / 2}(y)=O\left(y^{\sigma}\right)$ as $y \rightarrow 0$. If $f(x+i y)=$ $e(n x) y^{1 / 2} I_{s-1 / 2}(2 \pi|n| y)$, then $D f=s(s-1) f$. From these remarks it follows that $F_{n}(M z, s)=F_{n}(z, s)$ for all $M \in \Gamma$ and $D F_{n}(z, s)=s(s-1) F_{n}(z, s)$.

\section{§ 2. The Fourier Coefficients}

In this section it is shown that the Fourier coefficients of $F_{n}(z, s)$ are generalizations of the Fourier coefficients of analytic automorphic forms of integral weight. Again denote the general Kloostermann sum by $S(m, n ; c)$, and for simplicity let 


$$
M_{2 s-1}\left((m n)^{1 / 2} y\right)= \begin{cases}J_{2 s-1}\left(|m n|^{1 / 2} y\right), & m n>0 \\ I_{2 s-1}\left(|m n|^{1 / 2} y\right), & m n<0,\end{cases}
$$

THEOREM 1. Let $s=\sigma+i t^{\prime}, \sigma>1$, and $z=x+i y \in H$. Then

$$
F_{n}(z, s)=e(n x) y^{1 / 2} I_{s-1 / 2}(2 \pi|n| y)+\sum_{m=-\infty}^{\infty} b_{m}(y, s ; n) e(m x),
$$

where $b_{0}(y, s ; n)=B_{0}(s ; n) y^{1-s}$, and for $m \neq 0$,

$$
b_{m}(y, s ; n)=B_{m}(s ; n) y^{1 / 2} K_{s-1 / 2}(2 \pi|n| y) .
$$

Furthermore, $B_{0}(s ; n)=a_{n}(s) /(2 s-1)$, where $a_{n}(s)$ is the same coefficient as occurs in the Eisenstein series expansion, and

$$
B_{m}(s ; n)=2 \sum_{c>0} S(m, n ; c) c^{-1} M_{2 s-1}\left(4 \pi(m n)^{1 / 2} c\right) .
$$

Proof. We first note that $F_{n}(z, s)-e(n x) y^{1 / 2} I_{s-1 / 2}(2 \pi|n| y)$ is the sum over all pairs $(c, d)$, where $c>0$ and $\left(\begin{array}{ll}a & * \\ c & d\end{array}\right) \in \Gamma$, of

$$
e\left(n\left(a-(c x+d) /|c z+d|^{2}\right) / c\right)\left(y /|c z+d|^{2}\right)^{1 / 2} I_{s-1 / 2}\left(2 \pi|n| y /|c z+d|^{2}\right) .
$$

If we call this last function $e(n a / c) f(c x+d, y, c)$, and use the fact that $d=d^{\prime}+q c$, where $0 \leq d<c$ and $q$ is an integer, then

$$
\begin{aligned}
b_{m}(y, s) & =\int_{0}^{1}\left[F_{n}(z, s)-e(n x) y^{1 / 2} I_{s-1 / 2}(2 \pi|n| y)\right] e(-m x) d x \\
& =\sum_{c>0} \sum_{0 \leq d<c} e(n a / c) \sum_{q=-\infty}^{\infty} \int_{0}^{1} f(c x+d+q c, y, c) e(-m x) d x \\
& =\sum_{c>0} S(m, n ; c) \int_{-\infty}^{\infty} f(c x, y, c) e(-m x) d x \\
& =\sum_{c>0} S(m, n ; c) G(y, n, m, c) .
\end{aligned}
$$

If $m=0$, a change of variables shows

$$
\begin{aligned}
G(y, n, 0, c) & =|2 \pi n|^{-1 / 2} \int_{-\infty}^{\infty} \exp \left(i \alpha x /\left(x^{2}+y^{2}\right)\right)\left(\alpha y /\left(x^{2}+y^{2}\right)\right)^{1 / 2} I_{s-1 / 2}\left(\alpha y /\left(x^{2}+y^{2}\right)\right) \\
& =|2 \pi n|^{-1 / 2} G_{0}(y, \alpha), \quad \text { where } \alpha=2 \pi|n| / c^{2} .
\end{aligned}
$$

Similarly, if $m \neq 0$, then

$$
G\left(y|2 \pi m|^{-1}, n, m, c\right)=|2 \pi n|^{-1 / 2}|2 \pi m|^{-1} G_{1}(y, \beta),
$$

where 


$$
\begin{aligned}
G_{1}(y, \beta)= & \int_{-\infty}^{\infty} \exp \left(i x\left(1+\varepsilon \beta /\left(x^{2}+y^{2}\right)\right)\right) \\
& \times\left(\beta y /\left(x^{2}+y^{2}\right)\right)^{1 / 2} I_{s-1 / 2}\left(\beta y /\left(x^{2}+y^{2}\right)\right) d x,
\end{aligned}
$$

$\beta=4 \pi^{2}|m n| / c^{2}$ and $\varepsilon=m n /|m n|$.

Let

$$
w(t)=t^{1 / 2} I_{s-1 / 2}(t)
$$

and

$$
H(\alpha, x, y)=\exp \left(i \alpha x \varepsilon /\left(x^{2}+y^{2}\right)\right) w\left(\alpha y /\left(x^{2}+y^{2}\right)\right) .
$$

Since $w^{\prime \prime}(t)=w(t)\left(1+t^{-2}(s-1) s\right)$, a computation shows

$$
\alpha^{2} \frac{\partial^{2}}{\partial \alpha^{2}} H(\alpha, x, y)=s(s-1) H(\alpha, x, y)-i \varepsilon \alpha \frac{\partial}{\partial x} H(\alpha, x, y),
$$

if $\varepsilon^{2}=1$. Thus for $j=0,1$,

$$
G_{j}(y, \alpha)=\int_{-\infty}^{\infty} e^{i j x} H(\alpha, x, y) d x
$$

satisfies

$$
\alpha^{2} \frac{\partial^{2} G}{\partial \alpha^{2}}+[\varepsilon j \alpha-s(s-1)] G=0
$$

The formalities of differentiation under the integral and partial integration are easily justified for $\operatorname{Re} s>1$. Since $G_{j}(y, 0)=0$, it follows that

$$
G_{0}(y, \alpha)=G_{0}(y) \alpha^{s}
$$

and

$$
G_{1}(y, \alpha)=G_{1}(y) \alpha^{1 / 2} M_{2 s-1}\left(2(\varepsilon \alpha)^{1 / 2}\right)
$$

Now,

$$
\begin{aligned}
G_{0}(y) & =\lim _{\alpha \rightarrow 0} \alpha^{-s} G_{0}(y, \alpha) \\
& =2^{1 / 2-s}\left(\Gamma\left(s+\frac{1}{2}\right)\right)^{-1} \int_{-\infty}^{\infty} y^{s}\left(x^{2}+y^{2}\right)^{-s} d x \\
& =(2 \pi)^{1 / 2}(2 y)^{1-s} /(2 s-1) \Gamma(s) .
\end{aligned}
$$

Also, 


$$
\begin{aligned}
G_{1}(y) & =\lim _{\alpha \rightarrow 0} \Gamma(2 s) \alpha^{-s} G_{1}(y, \alpha) \\
& =2^{1 / 2-s} \Gamma(2 s)\left(\Gamma\left(s+\frac{1}{2}\right)\right)^{-1} y^{s} \int_{-\infty}^{\infty} e^{i x}\left(x^{2}+y^{2}\right)^{-s} d x \\
& =y^{1 / 2} K_{s-1 / 2}(y)
\end{aligned}
$$

This last equation follows from Basset's formula [7, p. 172]. Combining the formulae proved above settles the validity of the theorem.

COROLlaRY. Suppose $s$ is an integer larger than 1 . If $n>0$ and $G_{-2 s}(z, n)$ is the Poincaré series $\sum_{M \in \Gamma_{\infty} \backslash \Gamma} e(n M z)(c z+d)^{-2 s}$, then

$$
G_{-2 s}(z, n)=e(n z)+\pi(-1)^{s} \sum_{m=1}^{\infty}(m / n)^{s-1 / 2} B_{m}(s ; n) e(m z) .
$$

If $f$ is an analytic automorphic form for $\Gamma$ of dimension $2 s-2$, that is, of weight $1-s$, with principal part $\sum_{n=1}^{N} a_{n} e(-n z)$ at $\infty$, then $f(z)=\sum_{n=1}^{N} a_{n} g_{n}(z)$, where

$$
\begin{aligned}
g_{n}(z)= & e(-n z)+|\pi n|^{s-1 / 2} B_{0}(s ;-n) / \Gamma\left(s-\frac{\hat{i}}{2}\right) \\
& -\pi(-1)^{s} \sum_{m=1}^{\infty}(n / m)^{s-1 / 2} B_{m}(s ;-n) e(m z) .
\end{aligned}
$$

Proof. The Fourier expansions of these functions are derived in Chapters VIII and IX of [5], and this corollary is a restatement of those results.

\section{§3. The Analytic Continuation}

As we now show, $F_{n}(z, s)$ has a meromorphic continuation to the entire $s$-plane with poles located by the eigenvalues for $D$ and the poles of $E(z, s)$. The continuation is another consequence of the theory developed in [6].

Let $P_{\nu}$ and $Q_{\nu}$ be the Legendre functions of the first and second kinds, respectively. We shall be concerned with the point-pair invariant $k_{s}\left(z, z^{\prime}\right)=(1 / 2 \pi) Q_{s-1}\left(\cosh d\left(z, z^{\prime}\right)\right)$.

A point-pair invariant is a function $k\left(z, z^{\prime}\right)$ on $H \times H$ such that $k\left(T z, T z^{\prime}\right)=k\left(z, z^{\prime}\right)$ for all $T \in S L(2, R)$; hence, $k\left(z, z^{\prime}\right)$ is a function of $d\left(z, z^{\prime}\right)$. Each point-pair invariant defines an invariant integral operator

$$
L f(z)=\int_{H} k\left(z, z^{\prime}\right) f\left(z^{\prime}\right) d z^{\prime} .
$$


If $u$ is a function on $H$ such that $D u+\left(1 / 4+r^{2}\right) u=0$ and if $L u$ is defined, then $L u=h(r) u$, where $h$ is a function depending only on $k$. The linear operation $k \mapsto h$ is sometimes called the Selberg transform of $k$; see $[4, \S 5.3]$. For computational purposes we now show that this transform is related to the Mehler transform [1, p. 175].

THEOREM 2. Suppose that $k\left(z, z^{\prime}\right)=k\left(\cosh d\left(z, z^{\prime}\right)\right), k(t)$ is continuous for $1<t<\infty$, and $\int_{1}^{\infty} t^{-1 / 2}|k(t)| d t<\infty$. The Selberg transform is

$$
h(r)=2 \pi \int_{1}^{\infty} k(t) P_{-1 / 2+i r}(t) d t \quad \text { for real } r,
$$

and

$$
k(t)=\frac{1}{2 \pi} \int_{0}^{\infty} P_{-1 / 2+i r}(t) r \tanh (\pi r) h(r) d r \quad \text { for } 1<t<\infty .
$$

Proof. If we put $u(x+i y)=y^{1 / 2+i r}$, then $D u+\left(1 / 4+r^{2}\right) u=0$. Thus,

$$
h(r)=\int_{H} k\left(i, z^{\prime}\right) u\left(z^{\prime}\right) d z^{\prime} .
$$

If we put $\operatorname{Re} z^{\prime}=\sinh \rho \sin \theta$ and $\operatorname{Im} z^{\prime}=\cosh \rho+\sinh \rho \cos \theta$, then

$$
h(r)=2 \int_{0}^{\infty} \int_{0}^{\pi} k(\cosh \rho) \sinh \rho[\cosh \rho+\sinh \rho \cos \theta]^{-1 / 2+i r} d \theta d \rho .
$$

An integral representation for $P_{-1 / 2+i r}(\cosh \rho)$ in $[1, p .156]$ shows this is equivalent to the formula for $h$ given in the theorem. The formula for $k$ now follows from Theorem III in [3].

COROLlaRY. If $k_{s}\left(z, z^{\prime}\right)=(1 / 2 \pi) Q_{s-1}\left(\cosh d\left(z, z^{\prime}\right)\right)$, then the Selberg transform of $k_{s}$ is

$$
h_{s}(r)=\left[\left(s-\frac{1}{2}\right)^{2}+r^{2}\right]^{-1} \quad \text { for } \operatorname{Re} s>\frac{1}{2} .
$$

Proof. As $t \rightarrow 1, Q_{s-1}(t)+\frac{1}{2} \log \frac{1}{2}(t-1)$ has a finite limit, and as $t \rightarrow \infty, Q_{s-1}(t)=O\left(t^{-\sigma}\right)$, for $\sigma=\operatorname{Re} s$. The corollary follows from the theorem and equation (4) in [1, p. 170].

We now consider the integral operator

$$
L f(z)=\int_{H} k\left(z, z^{\prime}\right) f\left(z^{\prime}\right) d z^{\prime}
$$

acting on $L^{2}(\mathscr{D})$. With suitable conditions on $k$, 


$$
\int_{H} k\left(z, z^{\prime}\right) f\left(z^{\prime}\right) d z^{\prime}=\int_{\mathscr{D}} K\left(z, z^{\prime}\right) f\left(z^{\prime}\right) d z^{\prime},
$$

where $K\left(z, z^{\prime}\right)=\sum_{M \in \Gamma} k\left(z, M z^{\prime}\right)$. In terms of the transform $h$, it is pointed out in [6] that the sum defining $K$ will converge absolutely and uniformly on compact subsets of $H \times H$ if $h$ is an even analytic function for $|\operatorname{Im} r|<1 / 2+\varepsilon$, where $\varepsilon>0$, and $h(r)=O\left(\left(1+|r|^{2}\right)^{-1-s}\right)$ in this strip. As Selberg also points out, this kernel $K\left(z, z^{\prime}\right)$ then has an eigenfunction expansion:

$$
\begin{aligned}
K\left(z, z^{\prime}\right)= & \sum_{j=1}^{\infty} h\left(r_{j}\right) u_{j}(z) u_{j}\left(z^{\prime}\right) \\
& +\frac{1}{4 \pi} \int_{-\infty}^{\infty} h(r) E\left(z, \frac{1}{2}+i r\right) E\left(z^{\prime}, \frac{1}{2}-i r\right) d r,
\end{aligned}
$$

where $\left\{u_{j}\right\}_{j=0}^{\infty}$ is a maximal orthonormal set of real eigenfunctions in $L^{2}(\mathscr{D})$, and $D u_{j}+\left(1 / 4+r_{j}^{2}\right) u_{j}=0$. This information leads to

THEOREM 3. Let $w$ and $s$ be complex numbers with real parts larger than 1 and let $z, z^{\prime} \in H$. Let $k_{s}\left(z, z^{\prime}\right)=(1 / 2 \pi) Q_{s-1}\left(\cosh d\left(z, z^{\prime}\right)\right)$ and $h_{s}(r)$ $=\left[(s-1 / 2)^{2}+r^{2}\right]^{-1}$. If $\left\{u_{j}\right\}_{j=0}^{\infty}$ is a maximal orthonormal set of real functions in $L^{2}(\mathscr{D})$ such that $D u_{j}+\left(1 / 4+r_{j}^{2}\right) u_{j}=0$, then

$$
\begin{aligned}
\sum_{M \in \Gamma}[ & \left.k_{s}\left(z, M z^{\prime}\right)-k_{w}\left(z, M z^{\prime}\right)\right] \\
= & \sum_{j=0}^{\infty}\left[h_{s}\left(r_{j}\right)-h_{w}\left(r_{j}\right)\right] u_{j}(z) u_{j}\left(z^{\prime}\right) \\
& \quad+\frac{1}{4 \pi} \int_{-\infty}^{\infty}\left[h_{s}(r)-h_{w}(r)\right] E\left(z, \frac{1}{2}+i r\right) E\left(z^{\prime}, \frac{1}{2}-i r\right) d r .
\end{aligned}
$$

Moreover, the sum and integral on the right side of the equation converge absolutely and uniformly on compact subsets of $H \times H$ and they are meromorphic in $s$ for $\operatorname{Re} s>1 / 2$ with poles at $1 / 2 \pm i r_{j}$.

Proof. Since

$$
h_{s}(r)-h_{w}(r)=(w-s)(w+s-1)\left[\left(s-\frac{1}{2}\right)^{2}+r^{2}\right]^{-1}\left[\left(w-\frac{1}{2}\right)^{2}+r^{2}\right]^{-1},
$$

the first statement of the theorem follows from the discussion preceding the theorem. To prove the convergence properties we first suppose that $w>s>1$. Since $\left[k_{s}\left(z, z^{\prime}\right)-k_{w}\left(z, z^{\prime}\right)\right]$ is continuous in $z$ and $z^{\prime}$, the discussion preceding the theorem shows that $\sum_{M \in \Gamma}\left[k_{s}(z, M z)-k_{w}(z, M z)\right]$ is continuous for $z \in H$. Since the eigenvalues $1 / 4+r_{j}^{2}$ are nonnegative, the 
functions

$$
\begin{aligned}
F_{N}(z)= & \sum_{j=0}^{N}\left[h_{s}\left(r_{j}\right)-h_{w}\left(r_{j}\right)\right]\left|u_{j}(z)\right|^{2} \\
& +\frac{1}{4 \pi} \int_{-N}^{N}\left[h_{s}(r)-h_{w}(r)\right]\left|E\left(z, \frac{1}{2}+i r\right)\right|^{2} d r
\end{aligned}
$$

form an increasing sequence of continuous functions which converges to the continuous function $\sum_{M \in \Gamma}\left[k_{s}(z, M z)-k_{w}(z, M z)\right]$. Hence, the convergence is uniform on compact subsets of $H$.

Now suppose $\operatorname{Re} w>1$ and $S$ is a compact subset of $\{s: \operatorname{Re} s>1 / 2$, $\left.s \neq 1 / 2+i r_{m}, m \neq j\right\}$. Then for all $s \in S$, there is a constant $A$, such that $\left|h_{s}(r)-h_{w}(r)\right| \leq A\left[h_{2}(r)-h_{3}(r)\right]$ for all real $r$ or all $r$ in $\left\{r_{m}: 0<i r_{m} \leq\right.$ $1 / 2, m \neq j$ \}. From this inequality and Schwarz's inequality, one easily sees that

$$
\sum_{\substack{m=0 \\ m \neq j}}^{\infty}\left[h_{s}\left(r_{m}\right)-\left(h_{w}\left(r_{m}\right)\right] u_{m}(z) u_{m}\left(z^{\prime}\right)\right.
$$

and

$$
\int_{-\infty}^{\infty}\left[h_{s}(r)-h_{w}(r)\right] E\left(z, \frac{1}{2}+i r\right) E\left(z^{\prime}, \frac{1}{2}-r\right) d r
$$

converge uniformly for $s \in S$ and $\left(z, z^{\prime}\right)$ in any compact subset of $H \times H$. Since each eigenvalue has finite multiplicity, each point $1 / 2+i r_{j}$ in the interval $(1 / 2,1]$ is a pole. This proves all statements in the theorem.

CoRollary. Let $G(s)=\sum_{M \in \Gamma}\left[k_{s}\left(z, M z^{\prime}\right)-k_{w}\left(z, M z^{\prime}\right)\right]$ for $\operatorname{Re} s>1$, $\operatorname{Re} w>1$, and $\left(z, z^{\prime}\right) \in H \times H$. Then $G$ has a meromorphic continuation to the entire plane and $G(s)-G(1-s)=E(z, s) E\left(z^{\prime}, 1-s\right) /(2 s-1)$. The poles of $G(s)$ in the region $1 / 2 \leq \operatorname{Re} s$ are at the points $1 / 2 \pm i r_{j}$ and the residue of $(2 s-1) G(s)$ at $1 / 2+i r_{j}$ is $u_{j}(z) u_{j}\left(z^{\prime}\right)$ if $r_{j} \neq 0$ and $2 u_{j}(z) u_{j}\left(z^{\prime}\right)$ if $r_{j}=0$.

Proof. Let $f(\xi, s)=h_{s}(i(1 / 2-\xi))-h_{w}(i(1 / 2-\xi))$. Then $f$ is analytic in $\xi$ except at $s, 1-s, w$, and $1-w$; it has the residue $(1-2 s)^{-1}$ at $s$ and $(2 w-1)^{-1}$ at $w$; and $f(\xi, s)=f(\xi, 1-s)$. From the theorem,

$$
\begin{aligned}
G(s)= & \sum_{j=0}^{\infty} f\left(\frac{1}{2}+i r_{j}, s\right) u_{j}(z) u_{j}\left(z^{\prime}\right) \\
& +\frac{1}{4 \pi i} \int_{1 / 2-i \infty}^{1 / 2+i \infty} f(\xi, s) E(z, \xi) E\left(z^{\prime}, 1-\xi\right) d \xi .
\end{aligned}
$$


It also follows from the theorem that the sum on the right is meromorphic in the $s$-plane with poles and residues as stated in the corollary. To effect the continuation of the integral, we alter the path of integration.

Let $C$ be a rectalinear path which starts at $1 / 2-i \infty$ and connects $1 / 2-i \infty, 1 / 2-i B, A-i B, A+i B, 1 / 2+i B$ and $1 / 2+i \infty$, where $A>\max \{\operatorname{Re} s, \operatorname{Re} w\}$ and $B$ is a positive number which is chosen so that no poles of the integrand lie on $C$. Now suppose $\left\{\alpha_{j}\right\}_{j=1}^{N}$ are the poles of $E(z, \xi) E\left(z^{\prime}, 1-\xi\right)$ for $1 / 2<\operatorname{Re} \xi \leq 1$ and $|\operatorname{Im} \xi|<B$. Then

$$
\begin{aligned}
& \frac{1}{4 \pi i} \int_{1 / 2-i \infty}^{1 / 2+i \infty} f(\xi, s) E(z, \xi) E\left(z^{\prime}, 1-\xi\right) d \xi \\
&= \frac{1}{2} E(z, s) E\left(z^{\prime}, 1-s\right) /(2 s-1)-\frac{1}{2} E(z, w) E\left(z^{\prime}, 1-w\right) /(2 w-1) \\
&+\frac{1}{4 \pi i} \int_{C} f(\xi, s) E(z, \xi) E\left(z^{\prime}, 1-\xi\right) d \xi \\
&-\frac{1}{2} \sum_{j=1}^{N} f\left(\alpha_{j}, s\right) \operatorname{Res}_{\xi=\alpha_{j}} E(z, \xi) E\left(z^{\prime}, 1-\xi\right) .
\end{aligned}
$$

The right side is a meromorphic function $g(s)$ for $|\operatorname{Im} s|<B$ and $|\operatorname{Re} s-1 / 2|<A-1 / 2$, and in this region

$$
g(s)-g(1-s)=E(z, s) E\left(z^{\prime}, 1-s\right) /(2 s-1) .
$$

Since $A$ and $B$ can be arbitrarily large, the corollary is proved.

The connection between the functions $F_{n}(z, s)$ and the kernel considered in the previous theorem will now be shown.

TheOREM 4: Suppose $\operatorname{Re} s>1, \operatorname{Re} w>1$, and $\left(z, z^{\prime}\right) \in H \times H$. Let

$$
G\left(z, z^{\prime}, s, w\right)=\sum_{M \in \Gamma}\left[k_{s}\left(z, M z^{\prime}\right)-k_{w}\left(z, M z^{\prime}\right)\right],
$$

where $k\left(z, z^{\prime}\right)=(1 / 2 \pi) Q_{s-1}\left(\cosh d\left(z, z^{\prime}\right)\right)$. If $y>\max \left\{\operatorname{Im} M z^{\prime}: M \in \Gamma\right\}$, then the -nth Fourier coefficient

$$
\int_{0}^{1} G\left(x+i y, z^{\prime}, s, w\right) e(n x) d x
$$

is $y^{1-s} E\left(z^{\prime}, s\right) /(2 s-1)-y^{1-w} E\left(z^{\prime}, w\right) /(2 w-1)$ if $n=0$, and

$$
y^{1 / 2}\left[K_{s-1 / 2}(2 \pi|n| y) F_{n}\left(z^{\prime}, s\right)-K_{w-1 / 2}(2 \pi|n| y) F_{n}\left(z^{\prime}, w\right)\right]
$$

if $n \neq 0$.

Proof. Suppose $k\left(z, z^{\prime}\right)=k_{s}\left(z, z^{\prime}\right)-k_{w}\left(z, z^{\prime}\right)$. Then because the sum 
defining $G$ converges absolutely and uniformly,

$$
\begin{aligned}
\int_{0}^{1} G\left(x+i y, z^{\prime}, s, w\right) e(n x) d x & =\sum_{M \in \Gamma_{\infty} \backslash \Gamma} \sum_{m=-\infty}^{\infty} \int_{0}^{1} k\left(x+m+i y, M z^{\prime}\right) e(n x) d x \\
& =\sum_{M \in \Gamma_{\infty} / \Gamma} \int_{-\infty}^{\infty} k\left(x+i y, M z^{\prime}\right) e(n x) d x
\end{aligned}
$$

Suppose $M z^{\prime}=u+i v$. Then

$$
\begin{aligned}
\cosh d\left(z, M z^{\prime}\right) & =1+\left[(x-u)^{2}+(y-v)^{2}\right] / 2 y v \\
& =\left[(x-u)^{2}+y^{2}+v^{2}\right] / 2 y v .
\end{aligned}
$$

Thus we must evaluate

$$
\int_{-\infty}^{\infty} Q_{s-1}\left(\left(x^{2}+y^{2}+v^{2}\right) / 2 y v\right) e(n(x+u)) d x .
$$

From equation (47) in [2, p. 49], we have

$$
Q_{s-1}\left(\left(x^{2}+y^{2}+v^{2}\right) / 2 y v\right)=(y v)^{1 / 2} \int_{-\infty}^{\infty} K_{s-1 / 2}(y|t|) I_{s-1 / 2}(v|t|) e^{i t x} d t
$$

for $y>v>0$ and $\operatorname{Re} s>0$. By the Fourier inversion formula, the integral $(*)$ is $2 \pi e(n u)(y v)^{1 / 2} K_{s-1 / 2}(2 \pi|n| y) I_{s-1 / 2}(2 \pi|n| v)$ if $n \neq 0$ and $y>v>0$ and $\operatorname{Re} s>0$. Since $(*)$ is continuous in $n$, we let $n \rightarrow 0$ and find that

$$
\int_{-\infty}^{\infty} Q_{s-1}\left(\left(x^{2}+y^{2}+v^{2}\right) / 2 y v\right) d x=2 \pi y^{1-s} v^{s} /(2 s-1),
$$

if $y>v>0$ and $\operatorname{Re} s>1 / 2$. Thus the theorem follows from the definitions of $E(z, s)$ and $F_{n}(z, s)$.

THEOREM 5. Let $z^{\prime} \in H$ and $y>\max \{\operatorname{Im} M x: M \in \Gamma\}$. Suppose that the eigenfunctions referred to in Theorem 3 have the Fourier expansion $u_{j}(z)=\sum_{m=-\infty}^{\infty} c_{m}(j) y^{1 / 2} K_{i r_{j}}(2 \pi|m| y) e(m x)$. Then for $\operatorname{Re} s>1$ and $\operatorname{Re} w>1$,

$$
\begin{aligned}
& K_{s-1 / 2}(2 \pi|n| y) F_{n}\left(z^{\prime}, s\right)-K_{s-1 / 2}(2 \pi|n| y) F_{n}\left(z^{\prime}, w\right) \\
& =\sum_{j=0}^{\infty}\left[h_{s}\left(r_{j}\right)-h_{w}\left(r_{j}\right)\right] c_{-n}(j) y^{1 / 2} K_{i r_{j}}(2 \pi|n| y) u_{j}\left(z^{\prime}\right) \\
& \quad+\frac{1}{4 \pi} \int_{-\infty}^{\infty}\left[h_{s}(r)-h_{w}(r)\right] a_{-n}\left(\frac{1}{2}+i r\right) K_{i r}(2 \pi|n| y) E\left(z^{\prime}, \frac{1}{2}-i r\right) d r .
\end{aligned}
$$

Moreover, $F_{n}(z, s)$ has a meromorphic continuation to the entire s-plane; its only poles in the region $1 / 2 \leq \operatorname{Re} s$ are in $\left\{1 / 2 \pm i r_{j}\right\}_{j=0}^{\infty}$ and $s=1$ is not a pole; and finally, $F_{n}(z, s)-F_{n}(z, 1-s)=a_{-n}(s) E(z, 1-s) /(2 s-1)$. 
Proof. The first statement is a consequence of Theorems 3 and 4 . Theorem 4 and the corollary to Theorem 3 show that $K_{s-1 / 2}(2 \pi|n| y) F_{n}\left(z^{\prime}, s\right)$ has poles only in $\left\{1 / 2 \pm i r_{j}\right\}_{j=0}^{\infty}$ if $\operatorname{Re} s \geq 1 / 2$. But $K_{s-1 / 2}(2 \pi|n| y)$ has zeros only on the line $s=1 / 2+i r$, and for any such $s$ we may choose $y$ so that $K_{i r}(2 \pi|n| y)$ is not zero. The point $s=1$ is not a pole, for if $r_{j}=(1 / 2) i$, then $u_{j}$ is a harmonic automorphic form in $L^{2}(\mathscr{D})$. Thus $u_{j}$ is constant and $c_{n}(j)=0$ for $n \neq 0$, and hence the first statement of the theorem shows that no pole is at $s=1$. The functional equation follows from the corollary to Theorem 3 .

To conclude this section, we point out that Theorems 1 and 5 show that there is a representation for $\sum_{c>0} c^{-1} S(m, n ; c) M_{2 s-1}\left(4 \pi(m n)^{1 / 2} / c\right)$ which has a meromorphic continuation to the entire $s$-plane. It is hoped that this representation will shed new light on the growth of the Fourier coefficients of modular forms.

\section{§4. The Fourier Coefficients of Analytic Automorphic Functions}

As an application of the preceding theory we give a construction for all automorphic functions and cusp forms of weight one.

THEOREM 6. Suppose $f$ is an analytic automorphic function with principal part $\sum_{n=1}^{N} a_{n} e(-n z)$ at $\infty$. Then $f(z)=a_{0}+\sum_{n=1}^{N} a_{n} F_{-n}(z, 1)$.

Proof. Theorem 5 implies that $F_{n}(z, 1)$ is a harmonic automorphic function and its Fourier expansion is given by Theorem 1 , where $B_{m}(1 ; n)$ $=\lim _{s \rightarrow 1+} B_{m}(s ; m)$. Thus $f(z)-\sum_{n=1}^{N} a_{n} F_{-n}(z, 1)$ is a bounded automorphic function in $\mathscr{D}$ which is also harmonic. Hence, it is constant, which proves the theorem.

THEOREM 7. For $n \geq 1$, let

$$
G_{n}(z)=e(n z)-\pi \sum_{m=1}^{\infty} B_{m}(1 ; n)|m / n|^{1 / 2} e(m z)
$$

Then $G_{n}$ is a cusp form of weight 1 and the set $\left\{G_{n}\right\}_{n=1}^{\infty}$ spans the space of such forms.

Proof. Using Theorems 1 and 5, we see that for $n \geq 1, F_{-n}(z, 1)$ is the sum of an analytic function $f_{n}$ and the complex conjugate of an analytic function $g_{n}$, where 


$$
\begin{aligned}
f_{n}(z)= & e(-n z) / 2 \pi|n|^{1 / 2}+B_{0}(1 ;-n) \\
& +\frac{1}{2} \sum_{m=1}^{\infty} B_{m}(1 ;-n) e(m z) /|m|^{1 / 2}
\end{aligned}
$$

and

$$
g_{n}(z)=-e(n z) / 2 \pi|n|^{1 / 2}+\frac{1}{2} \sum_{m=1}^{\infty}\left[B_{-m}(1 ;-n)\right]^{-} e(m z) /|m|^{1 / 2}
$$

Note that the conjugate of $B_{-m}(1 ;-n)$ is $B_{m}(1 ; n)$, since $\left[B_{-m}(\bar{s} ;-n)\right]^{-}$ $=B_{m}(s ; n)$ for $\operatorname{Re} s>1$. Thus $-i|n|^{1 / 2} G_{n}(z)=g_{n}^{\prime}(z)$.

If the operator $\partial / \partial x+i(\partial / \partial y)$ is applied to the equation $F_{-n}(M z, 1)$ $=F_{-n}(z, 1)$, the resulting equation is equivalent to $G_{n}(M z)(d / d z) M z=$ $G_{n}(z)$. Thus $G_{n}$ is a cusp form of weight 1 .

To show that $\left\{G_{n}\right\}$ is a spanning set for the space $\mathscr{C}$ of all cusp forms of weight 1 , we let $h$ be the dimension of the subspace spanned by $\left\{G_{n}\right\}_{n=1}^{\infty}$. Let $I_{N}$ be the space spanned by $\left\{F_{-n}(z, 1)\right\}_{n=1}^{N}$ and $A_{n}$ be the space of automorphic functions that are in $I_{N}$. For sufficiently large $N$, the Riemann-Roch theorem implies that $\operatorname{dim} I_{N} / A_{N}=\operatorname{dim} \mathscr{C}$. But our previous arguments show that $h \geq \operatorname{dim} I_{N} / A_{N}$. Thus $h=\operatorname{dim} \mathscr{C}$.

Q.E.D.

THEOREM 8. Let $\left\{G_{n}\right\}_{n=1}^{\infty}$ be the set of functions defined in Theorem 7 . Then $\sum_{n=1}^{N} a_{n} F_{-n}(z, 1)$ is an analytic automorphic function if and only if $\sum_{n=1}^{N} a_{n} n^{1 / 2} G_{n}(z) \equiv 0$.

Proof. As shown in the proof of Theorem 7, $\sum_{n=1}^{N} a_{n} F_{-n}(z, 1)$ is the sum of an analytic function $f$ and the conjugate of an analytic function $g$. It also follows from the proof of Theorem 7 that $g$ is constant if and only if $\sum_{n=1}^{N} a_{n} n^{1 / 2} G_{n}(z)=0$. Since $\bar{g}$ is analytic in $H$ if and only if $g$ is constant, the theorem is proved.

\section{BIBLIOGRAPHY}

[1] A. Erdélyi, et al., Higher Transcendental Functions, Vol. I, McGraw-Hill, New York, 1953.

[ 2 ] — Tables of Integral Transforms, Vol. I, McGraw-Hill, New York, 1954.

[ 3 ] V. A. Fok, On the representation of an arbitrary function by an integral involving Legendre's functions with a complex index, C.R. (Dokl.) Acad. Sci. USSR, 39 (1943), 253-256.

[4] T. Kubota, Elementary theory of Eisenstein series, Kodansha LTD., Tokyo, 1973.

[5] Joseph Lehner, Discontinuous groups and automorphic functions, American Mathematical Society, Providence, 1964. 
[6] A. Selberg, Harmonic analysis and discontinuous groups in weakly symmetric riemannian spaces with applications to Dirichlet series, J. Indian Math. Soc., 20 (1956), 47-87.

[7] G. N. Watson, A Treatise on the Theory of Bessel Functions, Second edition, Cambridge University Press, Cambridge, 1952.

Department of Mathematics

University of Maryland 